\title{
Neues aus der AOTrauma Deutschland
}

\author{
Michael J. Raschke, Karl-Heinz Frosch
}

Die AOTrauma Deutschland entwickelt sich nach der Umstrukturierung dynamisch weiter. Das Kerngeschäft Fortbildung, Forschungsförderung und persönliche Weiterentwicklung der Mitglieder steht unverändert im Fokus.

Mit der Satzungsänderung im Jahr 2013 hat die Deutsche AO sich den internationalen Strukturen der Aufteilung in die klinischen Divisionen Trauma, Spine, CMF und VET angeglichen, ohne dabei die Gemeinsamkeiten und Gemeinschaftlichkeit aufzugeben. Die AOTrauma Deutschland verzeichnete vor Kurzem das 400. Mitglied, was für die Attraktivität des neuen Konzepts spricht.

Neu sind auch speziell verantwortliche Personen für die Bereiche Forschung, Prof. Georg Duda, Berlin, und Community Development, Prof. Karl-Heinz Frosch, Hamburg. Beide werden für ihre jeweiligen Bereiche neue Akzente setzen.

\section{Fortbildung}

Im Bereich der Schulung waren im Jahr 2014 insgesamt 398 Referenten und 52 Instruktoren in Kursen und Seminaren aktiv. Einige davon an mehreren Events, sodass es insgesamt zu 695 Einsätzen als Referent oder Instruktor kam. Insgesamt 1975 Kursteilnehmer verteilten sich auf 73 Kurstage. Im aktuellen Jahr sind 31 Kurse, 14 Seminare und 4 weitere Schulungsveranstaltungen geplant.

Wir möchten daran erinnern, dass Faculty-Mitglieder (Referenten und Instruktoren der AOTrauma-Veranstaltungen) auch AOTrauma-Mitglied sein müssen. In Zukunft werden wir es ermöglichen, den Mitgliedsbeitrag im Rahmen der Speseneinreichung zu verrechnen.

OP-JOURNAL 2015; 31: 61-62

(c) Georg Thieme Verlag KG Stuttgart · New York DOI http://dx.doi.org/10.1055/s-0035-1545976

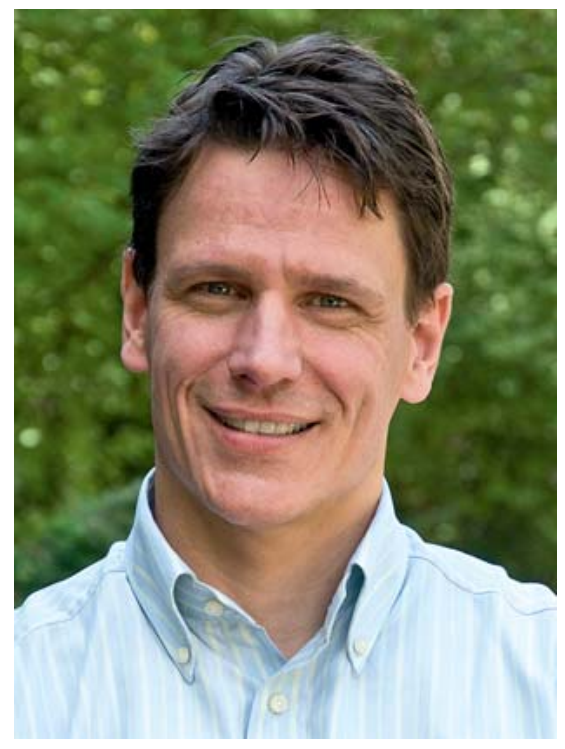

Abb. 1 Georg Duda.

Aktuell gibt es 23 AOTrauma-FellowshipZentren in Deutschland. Die Anzahl wird noch steigen, da weitere Kliniken sich beworben haben und diese Möglichkeit auch neuen Kliniken offen steht. So erlebt der ursprüngliche Gedanke und das Qualitätsmerkmal, AO-Klinik zu sein, eine Renaissance. Im Jahr 2014 kamen 66 Fellows nach Deutschland, davon 9 aus Europa, 34 aus Asien, 3 aus Lateinamerika, 5 aus Nordamerika und 15 aus dem Nahen Osten.

Ausbaufähig ist die Anzahl der deutschen Ärzte, die sich für Fellowships im Ausland bewerben. In 2014 wurden lediglich 2 deutsche Fellows gefördert, beide gingen in die USA. Hier werden wir in Zukunft verstärkt diese attraktive Förderung unseres Nachwuchses bewerben.

Hinweisen möchten wir in diesem $\mathrm{Zu}-$ sammenhang auf die geänderten AOTrauma-Fellowship-Kategorien. Neu ist das „Meet the Expert" Fellowship. Es richtet sich an erfahrene Chirurgen, die zur Verbesserung eines ganz speziellen fachlichen Gebiets für 2-4 Wochen zu

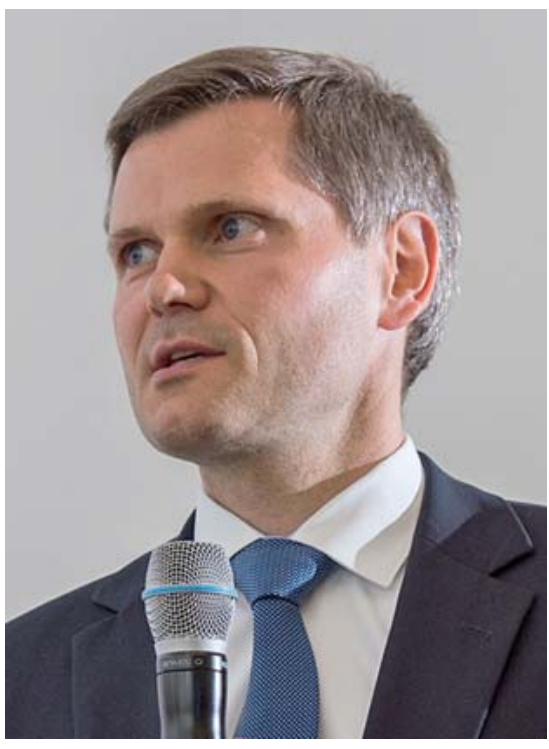

Abb. 2 Karl-Heinz Frosch.

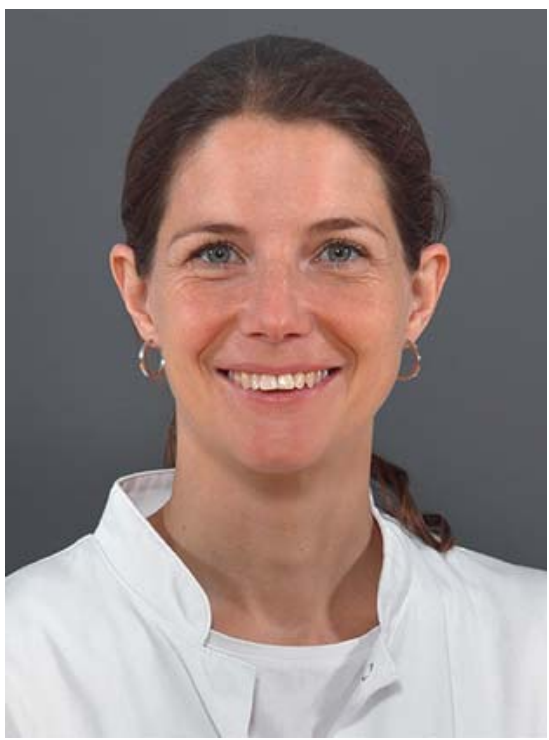

Abb. 3 PD Dr. Isabella Mehling.

einem entsprechenden Experten reisen wollen.

Das Reisestipendium 2015 wurde im Rahmen der Jahrestagung an Frau PD Dr. Isabella Mehling, BGU Frankfurt vergeben. Sie wird damit Prof. Jesse Jupiter in Boston 


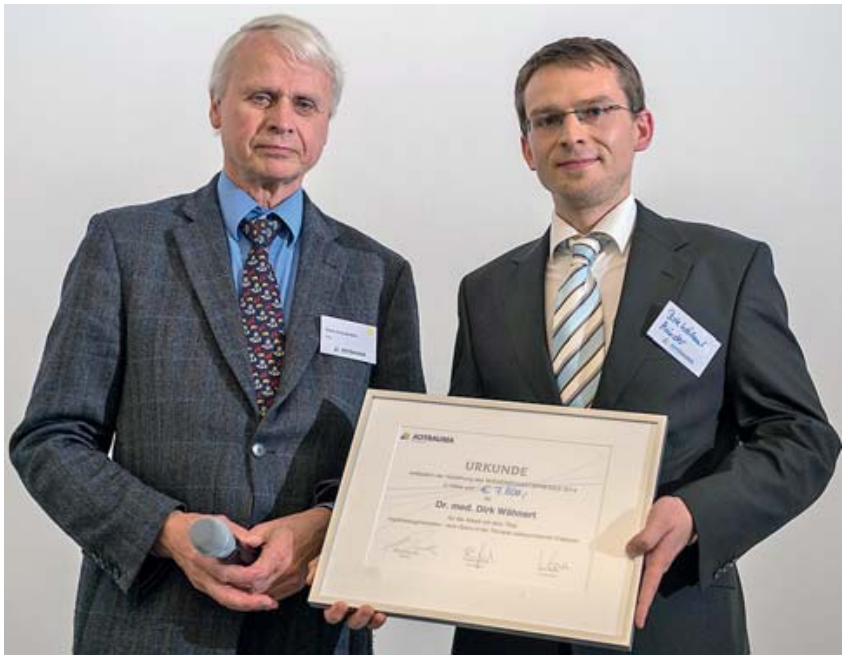

besuchen, um von seiner Expertise im Bereich Handchirurgie zu profitieren.

\section{Forschung \& Wissenschaftspreis}

Im Jahr 2014 wurden 6 Projekte gefördert mit einem Gesamtvolumen von $91000,-$ EUR.

Der Wissenschaftsspreis 2014 wurde an Dr. Dirk Wähnert, Münster, vergeben für seine Arbeit über „Implantataugmentation - eine Option in der Therapie osteoporotischer Frakturen“.
Abb. 4 Dr. Dirk Wähnert, Preisverleihung auf der Jahrestagung in Kiel.
Für den diesjährigen Preis werden noch Bewerbungen entgegengenommen. Der Preis wird am 20. Oktober auf dem AOTrauma Deutschland Symposium im Rahmen des DKOU vergeben. Einsendungen können noch zum bis 31. August geschickt werden an georg.duda@charite.de

Das Bewerbungsformular finden Sie unter www.aotrauma.org/deutschland

Auch für Forschungsanträge und Bewerbungen gilt: bitte vorher AOTrauma-Mitglied werden.

\section{Jahrestagung 2015}

Am 8./9. Mai fand in Tübingen, an dem Ort, an dem 45 Jahre zuvor die Absichtserklärung unterzeichnet wurde, die AO Deutschland zu gründen, die Jahrestagung mit über 100 Teilnehmern statt. Es war ein historisches Treffen mit dem ersten Vorsitzenden, dem heutigen Ehrenpräsidenten Siegfried Weller.

Auf der Mitgliederversammlung wurde das Geschäftsführende Präsidium für 3 weitere Jahre im Amt bestätigt. Dieses setzt sich zusammen aus: Michael Raschke (Präsident), Hans-Jörg Oestern (Past-Präsident), Florian Gebhard (VizePräsident) und Ulrich Stöckle (Schatzmeister). Aus dem Beirat standen 6 von 12 Posten zur Wahl.

$\mathrm{Zu}$ Ehrenmitgliedern wurden Prof. Hans Zwipp, Dresden, und Prof. Dankward Höntzsch, Tübingen, ernannt.

Weitere Informationen sind erhältlich unter www.aotrauma.org/Deutschland

Prof. Michael J. Raschke

Präsident, Münster

Prof. Karl-Heinz Frosch

Community Development, Hamburg

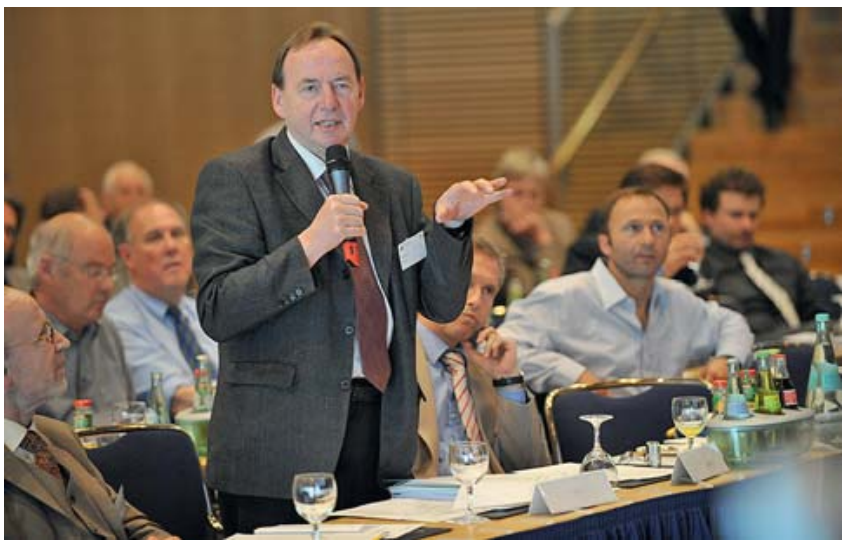

Abb. 5 Hans Zwipp.

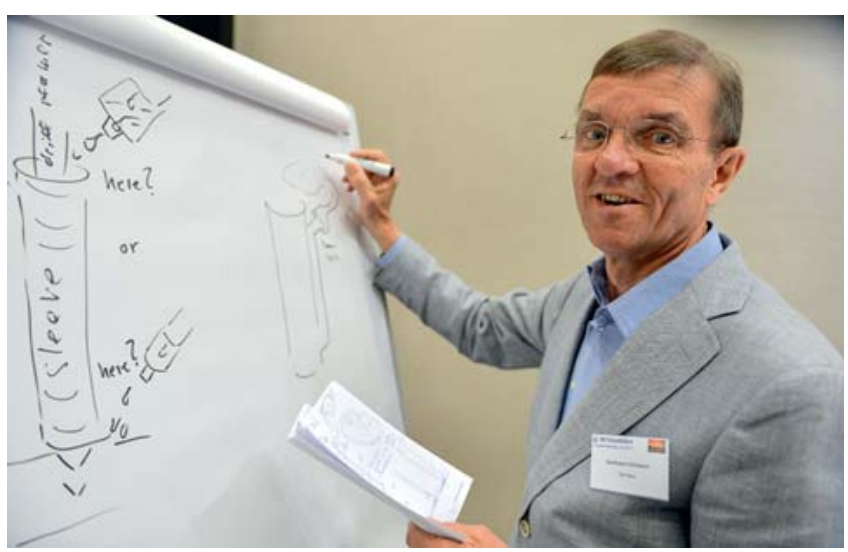

Abb. 6 Dankward Höntzsch. 